\title{
Persuasive Argumentation and Social Comparison as Determinants of Attitude Polarization
}

\author{
EUGENE BURNSTEIN
}

The University of Michigan

AND

Amiram VinOKuR

Tel-Aviv University

\begin{abstract}
Several of our studies indicate that persuasive-arguments theory by itself is an adequate explanation of polarization. Sanders and Baron (Journal of Experimental Social Psychology, 1977, 13, 303-314) criticize this research. More generally, they contend that both argumentation and comparison are involved, "with persuasive arguments facilitating the shifts motivated by social comparison." We feel that their critique is unconvincing. Relevant portions of the standard literature are reviewed to demonstrate that social comparison is neither a necessary nor sufficient condition for polarization. Finally, we speculate about how persuasive-arguments theory could be extended to argument-poor settings (e.g., Asch's line comparison situation).
\end{abstract}

Experience teaches us that the critical finding is a will-o'-the-wisp. Theoretical disputes in social psychology are settled, if ever, by the overall weight of evidence. So in the last analysis, whether persuasive argumentation alone or both persuasive argumentation and social comparison are needed to explain polarization will be decided on the basis of all the relevant data, which are massive, and not just on the few studies discussed by Sanders and Baron (1977). Our reading of the literature suggests that informational theories, such as persuasive-arguments, describe the immediate causes of polarization and by themselves offer a rough but reasonably complete account of the phenomenon. Theories based on normative influence, such as social comparison, deal with relatively remote processes which may lead to polarization on occasion, when they are mediated by persuasive argumentation.

Sanders and Baron disagree with us, and they are not alone. In a recent analysis of polarization research, Myers and Lamm (1976) conclude that

This paper was facilitated by Grant No. MH 29140-01 from the National Institute of Mental Health. Requests for reprints should be sent to Eugene Burnstein, Research Center for Group Dynamics, Institute for Social Research, University of Michigan, Ann Arbor Michigan 48106. 
"[although] the evidence for [informational theories such as persuasivearguments] is compelling . . . it also appears that group polarization is not fully explained by a passive process of cognitive learning. More dynamic processes of cognitive rehearsal and verbal commitment are also likely contributors." Their basis for disagreement is different from that stated in the Sanders and Baron paper. While we are concerned here mainly with the latter, it should become clear that the processes described in persuasive-arguments theory are quite "dynamic," if not mercurial. Indeed, it would be extremely difficult to analyze the construction and comprehension of arguments without assuming that the person acts on and transforms information (see also Anderson \& Graesser, 1976).

\section{THE PROBLEM}

The basic idea of persuasive-arguments theory is that when the person evaluates (or re-evaluates) alternative $\mathrm{X}$ relative to alternative $\mathrm{Y}$, he generates arguments, namely, ideas, images, or thoughts describing the attributes of $\mathrm{X}$ and $\mathrm{Y}$. This process assumes that there exists a culturally given pool of arguments speaking to each alternative. To judge the relative merits of these alternatives, the person samples (retrieves arguments) from this pool. Arguments may vary in availability (the probability of their coming to mind), direction (pro-X and pro- $\mathrm{Y}$ ), and persuasiveness. When the preponderance of arguments in the pool favors a particular alternative, the average prior attitude reflects the direction and magnitude of this preponderance. Further thought or discussion leads to polarization toward the alternative that initially elicits more and/or better arguments. Its magnitude will depend on whether the initial argument samples (a) overlap or (b) exhaust the larger pool. That is to say, polarization will be maximal when a person begins to rethink the issue and many arguments remain that have not yet come to mind, or when several individuals discuss the issue with each other and not all of them have thought of the same arguments.

Let us illustrate persuasive-arguments theory by a simple example. Consider a choice in which the culturally given pool contains six pro- $X$ arguments, $a, b, c, d, e$, and $f$, and three pro-Y arguments, $l, m$, and $n$. If three equally pro- $X$ individuals discuss the issue, one of several distinct outcomes would be predicted, depending on the distribution of arguments among members. Say all three of our discussants had thought of the same arguments. In this case, discussion would produce no change in their attitudes toward X. On the other hand, if $a, b$, and $m$ had come to mind in one; $c, d$, and $m$ in the second; and $e, f$, and $m$ in the third (i.e., if each has different pro- $X$ arguments, but the same pro- $Y$ arguments), then the discussion would produce marked polarization toward X. Finally, polarization toward $\mathrm{Y}$ would be predicted if one member had generated $a$, $b$, and $l$, another $a, b$, and $m$, and the third $a, b$, and $n$ (i.e., if each had 
initially thought of the same pro- $X$ but different pro- $Y$ arguments). Normally, individual argument samples are representative of the larger pool. Therefore, average prediscussion preferences can be estimated from the balance of pro- $X$ and pro- $Y$ arguments in the pool. Postdiscussion preferences can also be predicted if, in addition, we know the degree of overlap among individual argument samples (Vinokur \& Burnstein, 1974). An ingenious demonstration of the latter point was made recently by Kaplan and Miller (Note 2). They observed that when a series of arguments is learned prior to discussion, recall of a particular argument will depend on its position in the series (e.g., later arguments are remembered better than earlier ones). Thus, there should be less overlap in argument samples (i.e., a greater variety of arguments will be recalled during discussion) and consequently greater polarization when each member had learned the arguments in a different order rather than in the same order. This was in fact the case (also see Kaplan, Note 1, 1977).

Sanders and Baron propose that the information processes described by persuasive-arguments theory and the normative processes described by social comparison theory have complementary effects on polarization, "with persuasive-arguments facilitating the shifts motivated by social comparison." On balance, it seems to us that this proposal should be rejected on two grounds. First, it offers a needlessly complicated explanation of polarization. To illustrate, it is well known that polarization and convergence occur simultaneously. (In fact there are several "neutral" Choice-Dilemmas that produce convergence without polarization.) Specifically, the typical finding is that during discussion the most extreme member moves to a relatively moderate position while the next most extreme member hardly changes at all (e.g., Ferguson \& Vidmar, 1971). Although more than one version of social comparison theory is used to explain polarization (see below), all have difficulty in dealing with convergence. Usually a second process is posited. This seems unnecessary since persuasive-arguments by itself accounts for both effects on the basis of informational influence. According to persuasive-arguments theory, attitude polarization is fundamentally an informational phenomenon; normative influences are relatively remote and operate on polarization, if at all, through cognition. If a person is distressed or elated because others hold attitudes more extreme than his own and he is about to shift toward their position, this state of affairs will be reflected in ideation, that is, in the content of the argument sample, and can be taken into account at that point. In fact, we have observed somewhat to our surprise that when the attitudes of others are sharply opposed to the ideas they express, the latter determines the direction of polarization (Burnstein \& Vinokur, 1973, Experiment II). Hence, for the purpose of explaining polarization, social comparison theory may be excess baggage. The Sanders and Baron proposal is also difficult to justify in the light of past research. There is just 
too little evidence that social comparison processes have a direct affect on polarization. This large literature has been diligently reviewed by Myers and Lamm (1976). Thus, our discussion will focus on evidence that they touched on either lightly or not at all.

\section{PAST RESEARCH}

Social comparison theory, in one version or another (see the review by Pruitt, 1971), assumes: (a) a preference for alternative $X$ is more socially desirable than a preference for alternative $\mathrm{Y}$; (b) the person believes his own preference for $\mathrm{X}$ is at least as extreme as those of his peers (in Brown, 1965; Festinger, 1954; Jellison \& Riskind, 1970) or is too extreme to be acceptable (in Levinger \& Schneider, 1969; Pruitt, 1971); (c) upon learning this is untrue, he experiences distress (in the Brown, Festinger, and Jellison \& Riskind version) or relief (in the Levinger \& Schneider and Pruitt version); (d) either affective state causes the person to take a more extreme position which results in a decrease in distress (e.g., because according to Jellison \& Riskind he no longer appears less able than others) or an increase in satisfaction (e.g., because according to Pruitt he freely vents what was formally suppressed). These assumptions as well as those of persuasivearguments theory predict the conditions under which polarization will occur. Since these predictions often disagree, they can be pitted against each other in experiments. When this was done, we found that (i) if an individual could argue but not compare, polarization still occurred (Burnstein \& Vinokur, 1973); (ii) if he could compare but not openly argue, polarization vanished (Burnstein \& Vinokur, 1973, 1975) or was greatly attentuated (Burnstein, Vinokur, \& Trope, 1973); but (iii) even in attentuated form, polarization seemed to depend directly on tacit argumentation (Burnstein \& Vinokur, 1975). The latter experiment, however, did suggest that comparison processes can indirectly influence polarization; that is, information about others may guide the person in generating arguments. Sanders and Baron criticize each of our studies in some detail. Therefore, it will be convenient to discuss these results later when we reply to specifics of this critique. However, other findings also warrant attention.

In an unpublished study, we once instructed each subject to predict the distribution of choices of " 100 people like you" over the response scale. On the average subjects thought that about $30 \%$ of these persons would be more extreme than themselves. The assumption that an individual will be surprised, piqued, or elated to discover that one or two members of a five-person group prefer a more drastic course than he therefore seems unconvincing. Indeed, there is absolutely no evidence of a change in affect either when such a discovery is made or when the individual shifts toward a more extreme position. Yet these propositions about affective changes are 
easily tested. Hence, the absence of published research on this point is worrisome, especially since we do know that when the individual shifts toward a more extreme position he does not think that his posterior preference is any more socially desirable or adheres any more closely to widely cherished standards of conduct than his relatively moderate prior preference (Kahan, 1975). Moreover, diligent attempts to relate polarization to the belief that one is more extreme than his peers have been singularly unsuccessful (e.g., Lamm, Trommsdorff, \& Rost-Schaude, 1972). Our own concern about the issue led us to ask subjects on a postexperimental questionnaire to describe their state of mind upon learning that their preferences differed from those of other members (Burnstein \& Vinokur, 1975). Reports of distress or relief were rare and unrelated to polarization. However, polarization was correlated with the extent to which the person tried to explain the difference and with the amount of thought given to reasons why others made the choice they did (as well as with the number of such reasons he actually generated). Although the mental processes these people described are not those one would anticipate on the basis of a social comparison analysis, they are just what would be expected on the basis of persuasive-arguments theory.

A second line of evidence comes from several experiments in which members make their initial choices in terms of one feature of the decision task and then discuss either the same or another feature. For example, in testing a subjective expected utility (SEU) model with Choice-Dilemma items (the most widely used decision-task in polarization research), individuals indicated either (a) the minimal acceptable probability of success on the uncertain alternative or (b) the utility of the outcome associated with the certain alternative. In some conditions, both initial preferences and discussion had to do with the same feature (probability or utility); in other conditions, they involved different features. An individual ought to have a more clear-cut basis for comparing himself with others when preferences and discussion involve similar rather than different features. Thus, if social comparison processes are important, polarization should be attenuated under the latter conditions. In fact, no attenuation was observed (Burnstein, Miller, Vinokur, Katz, \& Crowley, 1971; DesJarlais, 1971; Vinokur, 1971). Furthermore, the SEU model assumes that extreme preferences are no more attractive per se than moderate ones (e.g., there is no utility for risk). Social comparison theory assumes that extreme preferences are more attractive. If the latter assumption were correct, SEU maximization would have been unable to account for the data. The fit between predicted and obtained preference, however, was extremely close, indicating that the social comparison assumption is unnecessary.

Finally, two sets of findings suggest most cogently that social comparison processes have little direct effect on polarization. The first 
concerns what the person thinks about and what he says in the polarization situation. Using the Choice-Dilemmas, Vinokur, Trope, and Burnstein (1975) performed a content analysis of the ideas that come to mind while a person formulates his attitude as well as those expressed during discussion. It was possible to identify several classes of arguments having to do with (a) the perceived utility of success and failure associated with the uncertain alternative and (b) the perceived utility of the certain alternative, both of which were called outcome utilities. In addition, since social comparison theory assumes that an extreme choice is in itself attractive, utilities associated with taking the "risky" or "cautious" action should exist independent of the utilities associated with the outcomes actually specified in Choice-Dilemma items. Arguments pertaining to these action utilities were also identified. (To illustrate, consider the choice between going to a highly prestigious university where a good proportion flunk out and a mediocre one where all receive their degree. In the face of this dilemma, two common arguments regarding outcome utilities are "he can always transfer to the easy school" and "he ought to be sure of getting his degree," the former, of course, supporting the choice of the uncertain alternative, the latter supporting the certain alternative. Examples of arguments concerned with action utilities are "life is no fun unless you take a chance" or "being cautious is a sign of wisdom.")

In fact, over $70 \%$ of the arguments generated by subjects in the above study (either privately or during discussion) were concerned with outcome utilities, whereas less than $10 \%$ were concerned with action utilities. Moreover, whereas outcome utilities were significantly correlated with polarization $(r=.63)$, action utilities were not $(r=.16)$. Finally, when outcome utilities (the persuasive arguments variable) and action utilities (the social comparison variable) were combined to predict polarization, the correlation was no higher $(r=.62)$ than when only outcome utilities were considered. If what a person thinks about, as well as what he says, reflects the relative significance of argumentation and social comparison, then these results suggest that the former process by itself has considerable impact on polarization, whereas the latter has very little.

While Sanders and Baron are less critical of the findings by Tesser and his co-workers than of ours (specifically, Burnstein \& Vinokur, 1975), both sets of results raise the same vexing problems (Sadler \& Tesser, 1973; Tesser \& Conlee, 1975; Tesser \& Cowan, in press; Tesser and Leone, 1976). Using a variety of attitude objects, the Tesser group discovered that if a moderately pro- $X$ individual simply thinks about the issue, this is sufficient to polarize his attitude toward X. In no instance did these effects depend on knowledge about the attitude of others; they occur without being "motivated by social comparison." In addition, the magnitude of polarization was shown to vary positively with (i) the number of arguments available to the person or his knowledge about the issue, (ii) the length of 
time he spends thinking about it, and negatively with (iii) the extent to which he is distracted from thinking. These three variables have special significance: In terms of social comparison theory, their relationships to polarization makes no sense at all. It is self-evident, however, that each in a different fashion govern the number of arguments likely to come to mind and that each operates just as persuasive-arguments theory would predict.

Our distraction experiment (Burnstein \& Vinokur, 1975) is similar to Tesser and Leone's (1976) except that in the former the person knew the preferences of others and in the latter he did not. Tesser used the probability of polarization rather than the amount of polarization as his dependent measure. When the comparable values are computed in our study, it appears that if the person was not distracted from generating arguments, the probability of polarization we observed (.48) was no greater than that observed by Tesser and Leone (.53), despite the fact that social comparison was possible in the former study but not in the latter. Furthermore, both experiments demonstrated that polarization could be made to decrease. Tesser and Leone found that this occurred to a similar degree when persuasive arguments were unavailable to the person (probability of polarization $=.33$ ) and when he was prevented from generating them (probability of polarization $=.25$ ). Moreover, the latter value is comparable to that obtained with the Burnstein and Vinokur distraction procedure (.19) where the individual was perfectly aware that others were more extreme than himself. These findings suggest (1) that social comparison does little to enhance or sustain polarization, (2) that polarization depends on a capacity to generate persuasive arguments, and (3) that this capacity is diminished when the person either is presented with an issue about which he has little knowledge or is prevented from thinking about the issue.

The polarizing effect of attention and thought on individual judgments has been observed in several different contexts. For instance, a large literature indicates that evaluations of visual or linguistic stimuli polarize as the frequency of exposure (and, thus, attention) to this material increases (e.g., Brickman, Redfield, Harrison, \& Crandall, 1977; Gush, 1976; Perlman \& Oscamp, 1971; Zajonc, 1968). Especially relevant are the studies comparing discussions and familiarization. Some years ago Bateson (1966) and Flanders and Thistlethwaite (1967) found that attitudes also polarized when individuals become familiar with the issue. Soon after, however, other studies appeared that were unable to obtain this effect (Bell \& Jamieson, 1970; Stokes, 1971; Teger, Pruitt, St. Jean, \& Haaland, 1970). The familiarization procedure essentially consists of having the subject think about and list arguments. On the face of it, this method seems quite similar to that of Tesser. The fact that Tesser has repeatedly found polarization to increase with thought, however, implies there must be some divergence in procedure heretofore overlooked. Not all of these studies 
provide the methodological information required for such an analysis. Nevertheless, an examination of those that do suggests two consistent and theoretically significant differences; namely, the instructional set for generating arguments and the time allowed for doing so. In Stokes (1971) as well as in Bell and Jamieson (1970) the instructions stress objectivity, thinking of arguments on "both sides" of the issue. By contrast, Bateson (1966), Flanders and Thistlethwaite (1967), and Tesser (1976) asked subjects to "use (their) imagination," to "make sure (they have) not overlooked some relevant information," to "read between the lines," or simply to "think about the issue until told to stop." Our conjecture is that the former instructions more so than the latter set the person to generate arguments which arrest polarization (e.g., an equal number of pro- $X$ and pro-Y arguments).

The second difference in procedure has to do with the time made available to the person for thinking about the issue before actually expressing a preference. If the person has a long time to generate arguments before indicating his initial position, he will exhaust the pool of available arguments. When he is again given the opportunity to think about the issue, no new arguments come to mind. As a result, his posterior attitude remains unchanged. More generally, polarization should decrease as the amount of thought given to a prior preference increases relative to the amount given to a posterior preference. Both in Bateson and in Tesser, the initial interval seems comparatively short. Bateson indicates that his subjects had about five times longer to think about their posterior position than their prior position. Tesser systematically varied the posterior interval. His initial interval, however, was always quite short; the subject had to give his "immediate reaction to the statement." Flanders and Thistlethwaite do not describe the relative length of these intervals in their experiment, nor do the studies which fail to obtain polarization, except for Bell and Jamieson (1970) who do say no time limit was imposed on the initial response. Presumably, this means the initial interval was determined by the slowest subject in the groups and, thus, on the average, was relatively long.

\section{A REPLY TO CRITICISM OF SPECIFIC EXPERIMENTS}

We feel that Sanders and Baron's criticisms of several of our studies are not well-founded. To begin with, their objections to many of our conclusions are based mainly on comparison of selected calls despite the absence of reliable main effects. For example, in our original study (Experiment I in Burnstein \& Vinokur, 1973), four of the five discussion items polarized (the fifth item approached but did not quite reach traditional levels of statistical significance). In the unpublished replication that Sanders and Baron cite, only one of the five polarized. Hence, a failure to 
replicate would be the most appropriate claim, and criticism of our study based upon the results pertaining to the single deviant item seems unjustified.

Furthermore, as Sanders and Baron note, the procedure used in this replication differed from the original (Experiment I in Burnstein \& Vinokur, 1973) in at least one significant respect. The earlier study attempted to demonstrate that persuasive argumentation was sufficient to produce a shift and that knowledge of others' actual attitudes was unnecessary. It found that when a member does not know whether others are arguing for their true position or its opposite, and the former in fact was the case, typical polarization effects are obtained. Subjects were not asked to infer the true position of others until the experiment was completed. Sanders and Baron, however, repeatedly asked subjects to draw such inference during the experiment. This procedure makes the inference task more salient than in the standard polarization study. Thus, it may distract subjects from thinking about the issue being discussed. In any case, persistent prodding does probably provide a better estimate of what the person is capable of inferring than our postexperimental questionnaire. Whether or not it is a more valid estimate of what he actually infers in the typical polarization situation is problematic. In our study (Burnstein \& Vinokur, 1973) we tried to create a situation in which inferences about another's attitude were not possible and the impossibility would be clear to subjects. Whatever may have been the case, when subjects are not repeatedly instructed to report the positions of others, we were able to determine that such inferences had no effect on posterior choices:

Internal analysis of the shifts produced by the subjects who correctly inferred the relationship between initial and advocated choice (a situation comparable to that of subjects in the typical polarization study) showed that they were not different from the shifts produced by the other subjects. In all cases the shifts of the correct subjects were in the same direction as the shifts of the other subjects, and in no case was the difference statistically significant. Indeed, . . . contrary to what would be predicted by comparison theories, the shifts of the correct subjects were smaller in magnitude than the shifts of the other subjects (Burnstein \& Vinokur, 1973; p. 129).

Internal analysis based on post facto questionnaire responses are inevitably ambiguous. This stricture applies to us as well as to Sanders and Baron in their replication. There is a difference, however. The internal analysis in Burnstein and Vinokur serves to emphasize that our conclusions do not depend on the person being able to guess correctly the position of others despite the experimental manipulation. But the conclusions themselves are based on main effects. Nevertheless, in order to be more certain about the consequence of guessing the position of others, we must await an experiment in which this is systematically varied. Still, it should be noted that several years ago a flurry of studies were reported in which group members learned about each other's position and 
then indicated their posterior preference without discussion. There was no need to guess or infer. If polarization occurred under these conditions, social comparison must be a sufficient cause. In many cases (Bell \& Jamieson, 1970; Clark, Crockett, \& Archer, 1971: St. Jean, 1970; St. Jean \& Percival, Note 3; Wallach \& Kogan, 1965) no polarization was observed. The remainder (Clark \& Willems, 1969; Teger \& Pruitt, 1967; Stokes, 1971) found an attenuated effect. These results were among the first to suggest that social comparison was not a sufficient cause and that an important mediating process was being overlooked.

Experiment II of this same article (Burnstein \& Vinokur, 1973) suggested that if a member had to argue for the "mirror-image" of his initial position-thus it should be difficult to muster persuasive argumentspolarization will not occur, even though the "mirror-image" rule was known and another's real attitude could be inferred. In fact, no risky item polarized; only when combined did they evidence a marginally significant shift toward risk. Similarly, no cautious item polarized; when combined, however, a reliable but atypical shift (toward risk) appeared. It was concluded that knowledge of others' attitudes was insufficient to motivate the typical polarization effect. Sanders and Baron say these results are "open to the alternative hypothesis (that) the weak and atypical shifts (the former referring to the marginal effect with risky items, the latter to the more robust effect with cautious items) . . . were due to pitting two active processes (argumentation and comparison) against one another." Let us follow their "pitting against" hypothesis to its logical conclusion. Our subjects were initially somewhat risky on the risky items and, given the "mirror-image" rule, tended to have to argue for caution. Because there was a whiff of polarization toward risk, Sanders and Baron must assume that comparison processes were slightly stronger. On cautious items these same subjects had to argue for risk. Here, an atypical shift toward the uncertain (risky) alternative was observed. If the "pitting against" hypothesis is to account for this effect, another assumption must now be made which is exactly the opposite of the one needed to explain marginal polarization on the risky item; namely, on the cautious items persuasive argumentation was stronger than comparison processes. Such reasoning is unconvincing. ${ }^{1}$

In Burnstein, Vinokur, and Trope (1973) individuals learned about choices of others and arguments for these choices before indicating their own preference. Both the number of choices and the number of arguments were varied orthogonally so that individuals in one condition received many choices and few arguments; in another condition, few choices and

\footnotetext{
${ }^{1}$ In criticizing this study, Sanders and Baron point out that self-persuasion through role-playing could have contributed to the results obtained. However, to the extent that such self-persuasion involves the self-generation of persuasive arguments, this possibility is not inconsistent with our position.
} 
many arguments, and so forth. The number of arguments had a reliable effect, but the number of choices did not. That polarization varied only with the number of arguments would seem to pose a real problem for social comparison theory. The pattern of shifts over conditions, however, suggested a cautious interpretation and led us to muse about how another's choice could, by stimulating thought, produce additional argumentation without group discussion: "Knowledge that other's choices are discrepant from his own may induce the person to reconstruct a line of reasoning (generate persuasive arguments) which he thinks could have produced (and thus would support) such choices" (Burnstein, Vinokur, \& Trope, 1973; p. 244). This conjecture was then tested in a later study (Burnstein \& Vinokur, 1975) which Sanders and Baron also find wanting. ${ }^{2}$

Their criticism, however, brings us back to the heart of the matter. Is the knowledge that one's own preference is relatively moderate sufficient to produce polarization? Or is there a more immediate cause, a process which intervenes between this knowledge and attitude change? Burnstein and Vinokur compared polarization across three conditions. In one, the person learned exactly how his position differed from those of others and was then encouraged to think further about the issue in question as well as to list all arguments which came to mind. In the second, the person learned about the difference between himself and others but was then distracted from thinking further about the issue. The third condition was identical to the first except the person received no information about another's position. Polarization occurred only in the first condition, its magnitude depending on the balance of arguments actually generated. We concluded that learning one was different is not sufficient to produce a shift in attitude; this knowledge was effective only to the extent that it moved the person to construct arguments explaining the difference. These findings also partly convinced Sanders and Baron. In their discussion of our study they agree that recognizing one's relative moderation is not enough, that there is a more immediate cause involved. Polarization they say, will not occur when the "cognitive work" which mediates the effect is blocked, as was the case

\footnotetext{
${ }^{2}$ In this context Sanders and Baron cite two interesting gambling experiments by Blascovich and colleagues (Blascovich \& Ginsburg, 1973; Blascovich, Ginsburg, \& Veach, 1975) in which no differences in bets are obtained between interacting (argumentation plus comparison) and coacting (comparison only) groups. In mentioning these findings they correctly note that persuasive-arguments predicts no polarization when there is nothing to discuss, namely, when it is impossible for the person to generate ideas. Sanders and Baron go on to assume that in the Blascovich situation arguments must be "sparse." According to persuasive-arguments, polarization should not occur. But polarization did occur. They conclude, therefore, it must be due to social comparison. To evaluate this line of reasoning all of Blascovich's major findings should be considered. The most important one is that appreciable polarization, the extraordinary increase in the size of bets, occurred under "alone" conditions, before the subjects had any opportunity to compare or to discuss. Ante hoc ergo propter hoc?
} 
in our distraction condition. The mediating processes that Sanders and Baron suggest, however, are on the spur of the moment (e.g. , "a desire not to look wishy-washy, which requires them to engage in rationalization before changing their position") and without theoretical or empirical support in the polarization literature. The processes described by persuasive-arguments theory, on the other hand, are reasonably well confirmed.

\section{EXTENDING PERSUASIVE-ARGUMENTS THEORY}

Two problems engage us at present. The first, which will be mentioned only briefly, has to do with persuasion. Up to now a persuasive argument has been defined simply as a statement judged to have a certain cogency. Recently we began to consider the theoretical basis for persuasiveness and ventured that this property depends on an argument's validity and originality. According to this formulation, an argument is an assertion or inference which follows from a line of reasoning. Validity would then refer to the soundness of the assertion-is it justified? The line of reasoning, which itself may involve a string of inferences, has as its premise the information available to the person when he begins to think about the issue. Ordinarily this preliminary information is contained in the way the issue is stated to the person, what he knows about the positions of others, and so forth. An argument's originality is defined by the number of steps in a line of reasoning. This is a rather important property since we assume that at each step, from premise to assertion, additional ideas are recruited and made available to the person. Therefore, when one either generates or comprehends a valid argument, the greater its originality, the more additional arguments are brought to mind. So far it has been possible to demonstrate crudely that if an argument is valid, both perceived and actual persuasiveness increase with originality (Vinokur \& Burnstein, in press).

We do not mean to imply that a valid line of reasoning must follow rules of formal logic. Sometimes, however, either little information is available with which to think about or discuss the choice (e.g., in Sheriff's autokinetic studies) or the information available appears to argue for a choice different from the one actually observed (e.g., in Asch's line judgment studies). Common sense might then suggest that informational processes are irrelevant. Nevertheless, there may be a mental representation of a line of reasoning even though one has difficulty describing the information it contains in words or images (see Natsoulas, 1970; Pylyshyn, 1973). Certainly it can be assumed that tacit argumentation occurs but is difficult to tap. One must then make derivations on the basis of this assumption that do have observable consequences. Which brings us somewhat awkardly to our second research problem: Can persuasivearguments theory be applied in situations where, on the face of it, 
argumentation seems gravely enfeebled? We will be especially concerned with cases in which normative influence is commonly thought to dominate information influence.

Sanders and Baron believe that the autokinetic judgment study in Baron and Roper (1976) is such a case. They reason that in this situation informational processes are virtually nonexistent because pithy assertions justifying a particular amount of movement seem unimaginable. Shifts in judgment, therefore, cannot be explained by argumentation and must be due to normative (comparison) processes. On the other hand, it is at least as plausible that by informing subjects that intelligent people give larger estimates of movement (their procedure for making the latter social desirable), Baron and Roper also provide them with a good argument for making such estimates: "I am intelligent, intelligent people make large estimates, therefore . . ." or "Intelligent people tend to be correct, I want to be correct, therefore. ..." Moreover, there are few persuasive arguments to the contrary since the actual amount of movement is totally ambiguous.

For our purposes the Asch line comparison procedure is more appropriate (Asch, 1956). The issue involved is completely unambiguous. In this case, one might expect arguments supporting an inaccurate judgment to be totally unconvincing and rare and those supporting the accurate judgment to be only slightly more numerous but immediately persuasive. We do not believe that undergraduates are so uncreative. In fact, the postexperimental interviews (Asch, 1956; Tuddenham \& McBride, 1959) reveal a surprisingly large number of not unpersuasive and even novel arguments which subjects attest to having affected their decision either to conform or to dissent (e.g., " "I figured the lines contained some sort of illusion that I was not subject to and they were" or "I wanted to make... (the experimenter's) results better.... (to avoid) the graph of results with a big dip in it."). Whether or not the rich ideation of Asch's subjects belies the common sense view that the line judgment task is "unthinkable" (and surely "undiscussable"), assume for the moment argumentation occurs. Let us now show that this assumption has testable implications that are, on occasion, counterintuitive.

In this context a person's prior and posterior choices of a comparison line refer to the judgments he makes before and after he is exposed to the preferences of others. The likelihood of a shift toward inaccuracy (or accuracy) depends on new ideas coming to mind during this interval. Persuasive-arguments theory assumes that whether a person's prior preference is strengthened, weakened, or remains unchanged, hinges on the degree to which (a) the argument he generated when making his original choice and (b) those he generates when induced to reconsider this choice are representative of the initial argument pool. If both are equally representative, then reconsideration will have no effect. If, however, a 
posterior sample is less representative than the prior one, preferences are likely to change, depending on the direction and extent of bias. Finally, the effects of a bias will be augmented if the argument sample generated while reconsidering is more exhaustive then the sample generated prior to initial choice.

A number of situational factors guide (bias) the person in generating arguments. For instance, knowing that a disagreement with the majority will be made public may induce the individual to consider ideas that would never have entered his mind if the disagreement had remained private. The two sources of bias to be examined here involve the knowledge of other's preference for a particular alternative and the monopolization of attention by this alternative. Both influence the representativeness and exhaustiveness of an argument sample. This means that they determine the likelihood of new ideas, and thus the likelihood of attitude change. We will illustrate these processes in terms of some well known results from Asch $(1951,1956)$ having to do with the size of a majority and the nonunamity of a majority.

An important and still puzzling finding is that the tendency to agree with a unaminous majority increased with the size of the majority but only up to the size of three (Asch, 1956). Hence, a unaminous majority need not be large to exert all of its potential influence. To begin, it is worth keeping in mind that individual choices are made known in series. Thus, being next to last, the person receives more information about the preferences of others and has a greater amount of time to generate arguments as majority size increases. The knowledge that another prefers, say, alternative $X$ rather than $Y$, focuses the person's attention on the former; the larger the majority, the more this alternative will monopolize attention and lead to the generation of a disproportionately large number of pro-X arguments. Therefore, the tendency to agree with a majority should continue to increase over a large range of sizes. Asch did not obtain such an effect and persuasive-arguments suggests why. At some point in the series the person will have exhausted the pool of pro-X arguments. From that point on, knowledge of another's preference will have no impact; the person simply will be unable to think of additional reasons for another choosing $X$. Whether this point occurs early or late in the series obviously depends on the number of pro-X arguments in the pool. In Asch's study the alternative preferred by the majority is unlikely to elicit many favorable arguments. Hence, it is no surprise that under these conditions the tendency to conform does not increase with the majority size beyond some relatively small value.

The influence of a majority can be considerably diminished if it is less than unanimous. The most common explanation of this effect assumes that the dissenter "liberates" the person to behave as he would really like. The implications of this analysis are rather different from those of persuasivearguments theory. For instance, the former suggests that once freed by the 
example of dissent a person will act correctly, as he would in the absence of an incorrect majority. From the point of view of persuasive argumentation, however, the minority merely guides attention away from the majority position, either in a correct or incorrect direction, depending on the minority's choice. Suppose the person is to select one of the three distinct alternatives, $\mathrm{X}, \mathrm{Y}$, or $\mathrm{Z}$; we know that $\mathrm{X}$ is correct (i.e., there exist many highly persuasive arguments in its favor), $\mathrm{Y}$ is moderately incorrect (i.e., there exists a medium number of supporting arguments), and $\mathrm{Z}$ is extremely incorrect (i.e., there exist few if any supporting arguments). Hence, when individuals choose alone, there is an overwhelming preference for $X$. Now, however, a majority selects $Z$ while a minority prefers $Y$. If the presence of a minority liberates the person to act as he normally would, then we should observe not merely a weakening of conformity (a decrease in the preference for $\mathrm{Z}$ ) but also a strengthening of correct behavior (an increase in the preference for $\mathrm{X}$ ). On the other hand, if a minority simply leads the person to think more extensively about its choice, no change in preference for the correct alternative would be observed. Instead, there should be a decrease in conformity coupled with an increased preference for the moderately incorrect alternative, $\mathrm{Y}$. We know of only one relevant study: Asch (1951) had a majority of seven choose the extremely incorrect line while a minority of one chose the moderately incorrect line. His findings were completely consistent with the persuasive-arguments analysis. There was a considerable shift in preference toward the moderately incorrect alternative but no change in the preference for the correct alternative.

\section{CONCLUSIONS AND SUMMARY}

According to Sanders and Baron "the question is not whether comparison is sufficient to produce choice shifts under all conditions but rather whether it is sufficient under the conditions in which choice shifts are typically (our italics) obtained." At least in respect to typical issues, which involve rich ideation, past research indicates that social comparison is neither a necessary nor a sufficient condition for polarization. At present, it seems this phenomenon can be adequately explained by persuasive argumentation alone. From a broader theoretical perspective, however, the relationship between persuasive-arguments and social comparison might be conceptualized as one in which the former deals with the more immediate determinants of polarization and the latter, with relatively remote determinants. Finally, it was suggested that persuasive-arguments could be extended beyond the typical polarization situtation to those involving ostensibly "undiscussable" issues, where argumentation seems virtually unimaginable (e.g., arguments for the incorrect line in Asch). Traditional analyses of social influence say that under such conditions changes in attitude must be due to normative processes. If this extension 
has merit, it suggests rather that social influence in general is essentially informational.

\section{REFERENCES}

Anderson, N. H., \& Graesser, C. C. An information integration analysis of attitude change in group discussion. Journal of Personality and Social Psychology, 1976, 34, 210-222.

Asch, S. E. Effects of group pressure on the modification and distortion of judgments. In $\mathrm{H}$. Geutzkow (Ed.), Groups, leadership, and men. Pittsburgh: Carnegie, 1951.

Asch, S. E. Studies of independence and conformities. Psychological Monographs, 1956, 70, No. 9 (Whole No. 416).

Baron, R. S., \& Roper, G. Reaffirmation of social comparison views of choice shifts: Averaging and extremity effects in an autokinetic situation. Journal of Personality and Social Psychology, 1976, 33, 521-530.

Bateson, N. Familiarization, group discussion, and risk-taking. Journal of Experimental Social Psychology, 1966, 2, 119-129.

Bell, P. R., \& Jamieson, B. D. Publicity of initial decisions and the risky shift phenomenon. Journal of Experimental Social Psychology, 1970, 6, 329-345.

Blascovich, J., \& Ginsburg, G. Blackjack and the risky shift. Sociometry, 1973, 36, 42-55.

Blascovich, J., Ginsburg, G., \& Veach, T. A pluralistic explanation of choice shifts on the risk dimenstion. Journal of Personality and Social Psychology, 1975, 31, 422-429.

Brickman, P., Redfield, J., Harrison, A. A., \& Crandall, R. Drive and predisposition as factors in attitudinal effects of mere exposure. Journal of Experimental Social Psychology, 1972, 8, 31-44.

Brown, R. Social Psychology. New York: Frec Press of Glencoc, 1965.

Burnstein, E., Miller, H., Vinokur, A., Katz, S., \& Crowley, J. The risky shift is eminently rational. Journal of Personality and Social Psychology, 1971, 20, 462-471.

Burnstein, E., \& Vinokur, A. Testing two classes of theories about group-induced shifts in individual choice. Journal of Experimental Social Psychology, 1973, 9, 123-137.

Burnstein, E., \& Vinokur, A. What a person thinks upon learning he has chosen differently from others: Nice evidence for the persuasive-arguments explanation of choice shifts. Journat of Experimental Social Psychotogy, 1975, 11, 412-426.

Burnstein, E., Vinokur, A., \& Trope, Y. Interpersonal comparison versus persuasive argumentation: A more direct test of alternative explanations for group induced shifts in individual choice. Journal of Experimental Social Psychology, 1973, 9, 236-245.

Clark, R. D., \& Willems, E. P. Where is the risky shift? Journal of Personality and Social Psychology, 1969, 13, 215-221.

Clark, R. D., Crockett, W. H., \& Archer, R. L. Risk as value hypothesis: The relationship between perception of self, others, and the risky shift. Journal of Personality and Social Psychology, 1971, 20, 425-429.

DesJarlais, D. Shifts to risk and reward. Psychological Reports, 1970, 26, 119-122.

Ferguson, D. A., \& Vidmar, N. Effects of group discussion on estimates of culturally appropriate risk levels. Journal of Personality and Social Psychology, 1971, 20, $436-445$.

Festinger. L. A theory of social comparison processes. Human Relations, 1954, 7, 117-140.

Flanders, J. P., \& Thistlethwaite, D. L. Effects of familiarization and group discussion upon risk-taking. Journal of Personality and Social Psychology, 1967, 5, 91-98.

Gush, J. E. Attitude formation and mere exposure: A non-artifactual explanation of empirical findings. Journal of Personality and Social Psychology, 1976, 33, 281-290.

Jellison, M., \& Riskind, J. A social comparison of abilities interpretation of risk taking behavior. Journal of Personality and Social Psychology, 1970, 15, 375-390.

Kahan, J. P. A subjective probability interpretation of the risky shift. Journal of Personality and Social Psychology, 1975, 31, 977-982. 
Kaplan, M. F. Judgment by juries. In M. F. Kaplan \& S. Schwartz (Eds.), Human judgment and decision-making in applied settings. New York: Academic Press, 1977.

Lamm, H., Trommsdorff, G., \& Rost-Schaude, E. Self-image, perception of peers' risks acceptance and risky shift. European Journal of Social Psychology, 1972, 2, 255-272.

Levinger, G., \& Schneider, D. J. A test of the "Risk is a Value" hypothesis. Journal of Personality and Social Psychology, 11, 165-169.

Myers, D. G., \& Lamm, H. The group polarization phenomenon. Psychological Bulletin, $1976,83,602-627$.

Natsoulas, T. Concerning introspective "knowledge." Psychological Bulletin, 1970, 73, 89-111.

Perlman, D., \& Oskamp, S. The effects of picture content and exposure frequency on evaluations of Negroes and whites. Journal of Experimental Social Psycology, 1971, 7, 503-514.

Pruitt, D. G. Choice shifts in group discussion: An introductory review. Journal of Personality and Social Psychology, 1971, 20, 339-360.

Pylyshyn, Z. W. What the mind's eye tells the mind's brain: A critique of mental imagery. Psychological Bulletin, 1973, 80, 1-24.

Sadler, O., \& Tesser, A. Some effects of salience and time upon interpersonal hostility and attraction during social isolation. Sociometry, 1973, 36, 99-112.

Sanders, G., \& Baron, R. S. Is social comparison irrelevant for producing choice shifts? Journal of Experimental Social Psychology, 1977, 13, 303-314.

St. Jean, R. Reformation of the value hypothesis in group risk taking. Proceeding of the 78 th Annual Convention of the American Psychological Association, 1970, 5, 339-340.

Stokes, J. P. Effects of familiarization and knowledge of others' odd choices on shifts to risk and caution. Journal of Personality and Social Psychology, 1971, 20, 407-412.

Teger, A. I., \& Pruitt, D. G. Components of group risk taking. Journal of Experimental Social Psychology, 1967, 3, 189-205.

Teger, A. I., Pruitt, D. G., St. Jean, R. \& Haaland, G. A. A re-examination of the familiarization hypothesis in group risk taking. Journal of Experimental Social Psychology, 1970, 6, 346-350.

Tesser, A. Attitude polarization as a function of thought and reality constraints. Journal of Research in Personality, 1976, 10, 183-194.

Tesser, A., \& Conlee, M. C. Some effects of time and thought on attitude polarization. Journal of Personality and Social Psychology, 1975, 31, 262-270.

Tesser, A., \& Cowan, C. L. Some effects of thought and number of cognitions on attitude change. Social Behavior and Personality, in press.

Tesser, A., \& Leone, C. Cognitive schema and thought as determinants of attitude change. Journal of Experimental Social Psychology, in press.

Tuddenham, R. D., \& McBride, P. D. The yielding experiment from the subject's point of view. Joumal of Personality, 1959, 27, 259-271.

Vinokur, A. Review and theoretical analysis of the effects of group processes upon individual and group decisions involving risk. Psychological Bulletin, 1971a, 76, 231-250.

Vinokur, A. Cognitive and affective processes influencing risk-taking in groups: An expected utility approach. Journal of Personlaity and Social Psychology, 1971b, 20, 472-486.

Vinokur, A., \& Burnstein, E. The effects of partially shared persuasive agruments on group induced shifts: A group problem solving approach. Journal of Personality and Social Psychology, 1974, 29, 305-315.

Vinokur, A., \& Burnstein, E. Novel argumentation and attitude change: The case of polarization following group discussion. European Journal of Social Psychology, in press.

Vinokur, A., Trope, Y., \& Burnstein, E. A decision-making analysis of persuasive argumentation and the choice-shift effect. Journal of Experimental Social Psychology, $1975,11,127-148$. 
Wallach, M. A., \& Kogan, N. The roles of information, discussion, and consensus in group risk taking. Journal of Experimental Social Psychology, 1965, 1, 1-19.

Zajonc, R. B. The attitudinal effects of mere exposure. Journal of Personality and Social Psychology, 1968, 9, 1-27.

\section{REFERENCE NOTES}

1. Kaplan, M. F. Group discussion effects in a modified jury decision paradigm: Information influences. Paper presented at the meeting of the Psychonomic Society, Denver, November 1976.

2. Kaplan, M. F., \& Miller, C. E. Juror judgments and discussion: Effects of variety of shared information on amount of polarization. Paper presented at the meeting of the Psychonomic Society, St. Louis, November 1976.

3. St. Jean, R., \& Percival, E. The effects of information control on shifts in risk taking. Paper presented at the Eastern Psychological Association, May 1973. 